\title{
Retrouver une vision (artificielle) grâce aux implants rétiniens
}

Un grand espoir repose aujourd'hui sur les implants rétiniens pour rendre une vision utilisable à des patients devenus aveugles. Différents protocoles de recherche sont actuellement en cours. Un implant récemment mis au point, I'Argus II de Second Sight, devrait obtenir le marquage CE d'ici la fin de l'année 2010.

\section{Pierre-Olivier BARALE \\ Saddek MOHAND-SAID \\ Sarah AYELLO-SCHEER \\ Jeanne HAÏDAR \\ Serge PICAUD \\ José Alain SAHEL \\ Centre d'investigation clinique \\ (Inserm $\mathrm{CIC} 503$ ) \\ CHNO des Quinze-Vingts, \\ Institut de la Vision, Paris \\ mohand@quinze-vingts.fr}

\section{Les implants rétiniens}

Les implants rétiniens s'adressent à des patients présentant une perte fonctionnelle des photorécepteurs entraînant une cécité ou quasi-cécité avec conservation d'un nerf optique sain permettant le transfert du signal aux aires visuelles du cerveau, comme les patients atteints de rétinopathie pigmentaire.

Le principe de ces implants est de stimuler par des électrodes les cellules neuronales rétiniennes (cellules ganglionnaires) résiduelles. Différentes études ont validé cette possibilité de stimulation et prouvé que des patients aveugles pouvaient percevoir des perceptions lumineuses par ces stimulations électriques. L'emplacement des électrodes, leur nombre, l'intensité et le site des stimulations (horizontales, verticales) ont permis à des patients de s'orienter vers une source lumineuse, de suivre des lignes au sol et de percevoir certains objets prédéfinis par contraste. Des résultats ont été obtenus chez I'homme par différentes équipes avec deux types d'implants : les implants sous-rétiniens (figure 1) avec des électrodes placées sous la rétine ( $\mathrm{Pr}$ Zrenner,

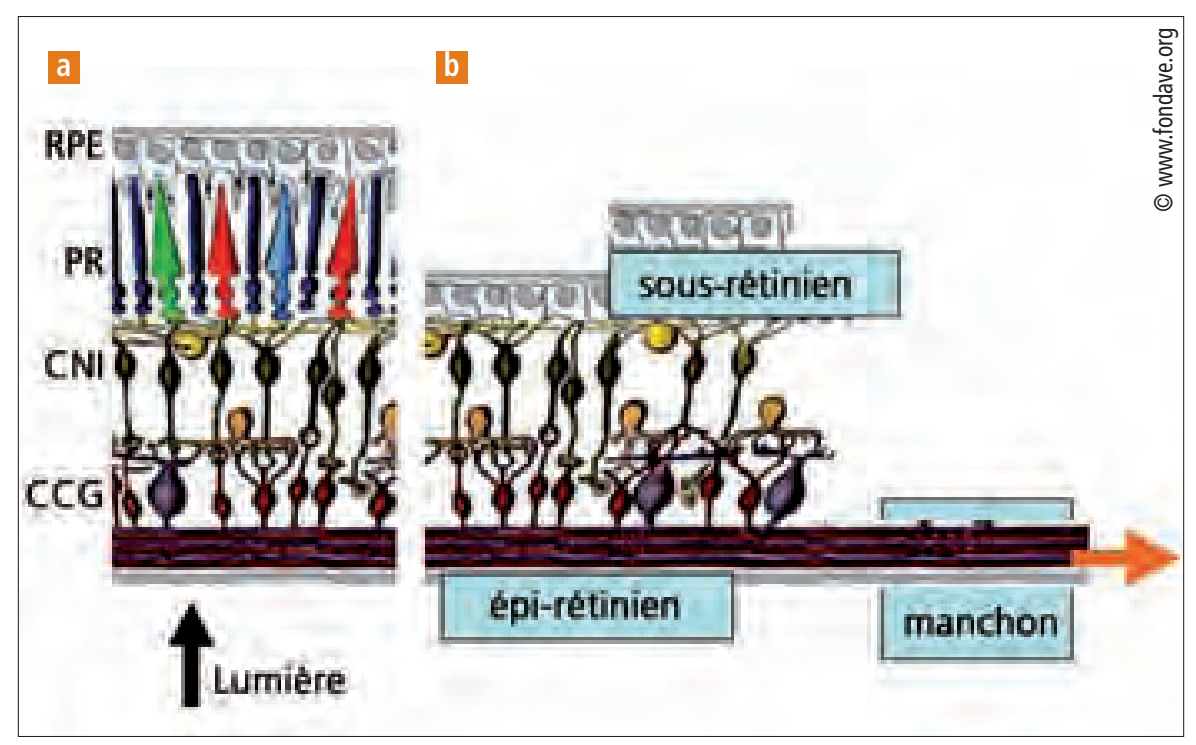

Allemagne) et les implants épi-rétiniens avec des électrodes placées en avant de la rétine ( $\mathrm{Pr}$ Humayun, États-Unis).

La taille et le nombre de phosphènes obtenus par les premiers systèmes (Argus I, 16 électrodes) ne permettaient pas une résolution spatiale importante, mais ils ont validé la tolérance et la faisabilité du principe.

\section{Combien de pixels faut-il pour "voir"?}

Aujourd'hui, les implants disponibles ont au plus 60 électrodes (et permettent donc des images ayant autant de points ou pixels), ce qui permet une vitesse de lecture de 20 à 35 mots par minute. Or, d'après les travaux du professeur Safran, à Genève, basés sur des simulations, il semble qu'un minimum de 600 points soit nécessaire pour lire un texte à une vitesse de 60 à 75 mots par minute. L'augmentation du nombre d'électrodes, de 16 à 60 pixels en quatre années, est une première étape mais
Figure 1. La rétine (a) est constituée de photorécepteurs $(\mathrm{PR})$, véritables photocapteurs biologiques, et d'un réseau neuronal en deux couches (couche nucléaire interne CNI, couche des cellules ganglionnaires (CG).

b. La perte des photorécepteurs laisse le réseau neuronal sans stimulation. L'objectif des implants rétiniens est de réintroduire une stimulation du réseau neuronal pour rétablir l'envoi d'images visuelles au cerveau par le nerf optique (flèche orange). Ces implants (rectangles bleus) peuvent être positionnés sous la rétine à la place des photorécepteurs (sous-rétinien), à la surface de la rétine du côté des cellules ganglionnaires (épi-rétinien) ou autour du manchon du nerf optique.

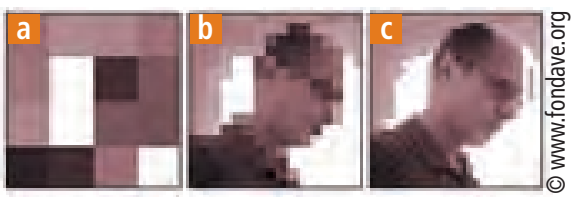

Figure 2. Images que percevraient des patients porteurs d'un implant rétinien à 16 (a), 256 (b) et 1000 électrodes (c) (images générées par l'Artificial Retinal Implant Vision Simulator développé par Wolfgang Fink au Visual and Autonomous Exploration Systems Research Laboratory, California Institute of Technology). 

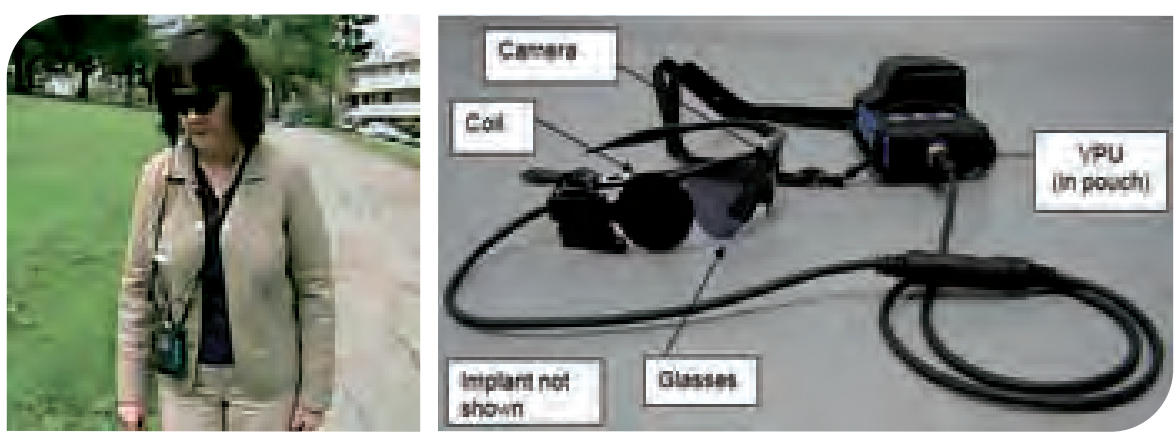

Figure 3. Patient portant le système Argus II et détail de celui-ci (Second Sight).

reste un des points clés à améliorer pour rendre la vision artificielle confortable (figure 2).

\section{Mise en pratique}

Depuis février 2008, quatre implantations épi-rétiniennes de seconde génération (Argus II, 60 électrodes) ont été effectuées par l'équipe du Pr Sahel ( $D^{r}$ Barale, Dr Ayello-Scheer, Dr MohandSaid) à I'hôpital des Quinze-Vingts (Paris), dans le cadre d'une étude internationale multicentrique (États-Unis, Mexique, Europe) financée par la société Second Sight*. Les patients ont ensuite été suivis régulièrement au Centre d'investigation clinique de l'hôpital pour apprendre à utiliser le dispositif, procéder à des examens cliniques et des tests visuels hebdomadaires.

L'implantépi-rétinien s'adresse à des patients présentant une rétinopathie pigmentaire évoluée (acuité visuelle maximale limitée à une perception lumineuse) ayant déjà eu une vision utile auparavant. Son principe est de transmettre l'information visuelle à la rétine via une caméra, un dispositif électronique et des électrodes posées sur la rétine.

Le principe de cet implant repose sur le passage du signal visuel par une caméra, qui le transmet à un boîtier le transformant en un signal transmis à un émetteur haute fréquence.

*Les auteurs n'ont aucun intérêt financier dans le produit.
Le signal est alors envoyé à un récepteur placé sur l'œil du patient qui le transmet aux électrodes posées sur la rétine.

Dans le cas de l'Argus II, le patient porte des lunettes sur lesquelles une caméra est fixée. Il s'agit d'une mini-caméra numérique qui envoie l'image numérique à un boîtier (processeur visuel miniature, VPU) porté dans une poche par le patient (figure 3). Ce boîtier convertit le signal numérique de l'image en un signal électrique radio, qui est alors émis vers le récepteur posé sur l'œil du patient. Ce récepteur est fixé par des points de suture en fil non résorbable sur la sclère de l'œil, et maintenu plus solidement par une bande de silicone (cerclage) placée autour de l'œil (figure 4). Il est relié par un câble à une plaque de 60 électrodes placée à la surface de la rétine, maintenue par un clou rétinien transrétino-scléral (figure 5).

Figure 4. Principe de l'implant rétinien (Second Sight).

1. Plaque d'électrodes épi-rétinienne,

2. Câble reliant au récepteur.

3. Récepteur sur la sclère.

Cette plaque est l'implant rétinien : c'est elle qui envoie le signal électrique à la rétine. Cette dernière le réceptionne par les cellules ganglionnaires qui le transmettent enfin au cerveau par les voies neuronales habituelles. On peut ainsi pallier au défaut de réception des cellules photoréceptrices rétiniennes malades.
Optique adaptative en microseopic

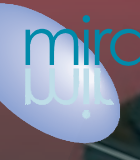

Le miroir déformable mirao 52-e, avec ou sans analyseur de surface d'onde HASO, avec nos logieiels CASAO OU GENAO, offre la meilleure capacité de correction et permet d'améliorer la résolution de l'image de manière spectaculaire.

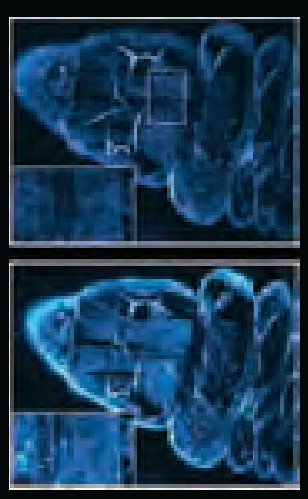

En haut, imagge d'une larve de drosophile en mieroscopie THG non-corrigée. En bas, image du même échantillon corrigée avec l'optique adaptative. Images de E. Beaurepaaire, D. Débarce et N. Olivier, Eeole Polytechnique, LOB

Venez nous rencontrer à PRI/OPTO 2010, du 26 au 28 octobre au Parc Floral de Paris sur le stand J9.

Pour plus dinformations, appelez +33 (0)1 6486 15 60, ou visitez: imagine-optic.80m

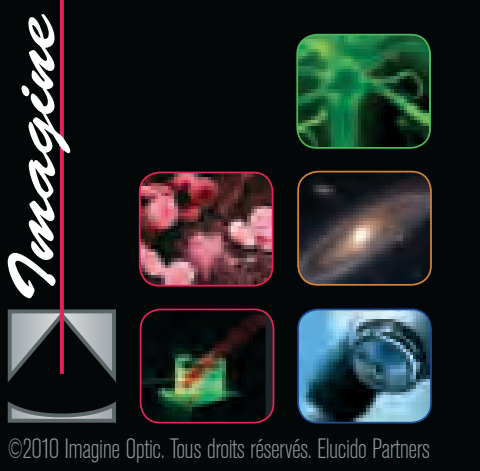


Figure 5. Image du fond d'œil d'un patient montrant le positionnement de la plaque d'électrodes fixée en transrétino-scléral (implant rétinien Argus II).
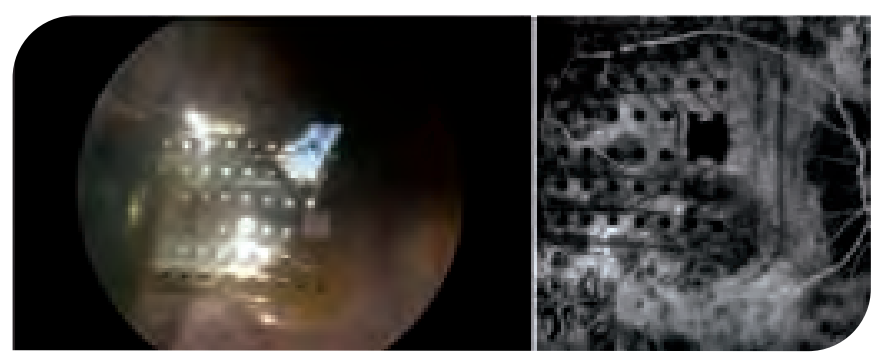

Une fois le dispositif en place, un très important travail de suivi est nécessaire pour optimiser le codage de l'image avec l'aide active du patient qui doit, lui aussi, apprendre à utiliser ces nouvelles informations visuelles.

\section{Résultats et voies d'optimisation}

Les résultats de cette étude ne sont pas encore publiés, mais les données préliminaires enregistrées sont encourageantes, tant sur la sécurité (la faisabilité et l'innocuité de la procédure d'implantation ont été quasiment validées dans tous les centres et les effets indésirables sont similaires à ceux d'autres procédures de chirurgie de la rétine courantes) que d'un point de vue fonctionnel.

En effet, les performances visuelles testées dans le cadre strict du protocole sont très satisfaisantes et les capacités de discrimination escomptées semblent atteintes. Les patients peuvent à nouveau suivre sans interruption un tracé au sol ou encore, identifier et atteindre aisément une ouverture (porte ou fenêtre) dans un mur.

Ainsi, les résultats sur l'ensemble des patients de l'étude montrent que $96 \%$ des patients présentent une réelle amélioration de leur acuité visuelle après plusieurs séances d'entraînement et de rééducation. Les "meilleurs patients" $(22 \%)$ atteignent même une acuité visuelle que l'on peut estimer être entre " mouvement de la main » et " compter les doigts » et peuvent également identifier des lettres et lire des mots de quatre lettres présentés sur un écran d'ordinateur.

D'autres performances visuelles, dont l'évaluation n'était pas prévue dans le protocole initial de l'étude, ont été rapportées spontanément par certains patients, comme la capacité retrouvée de détecter des mouvements de personnes à travers leur fenêtre ou de noter des changements de disposition de certains éléments de décor de leur environnement domestique.

Il est important de préciser que ces précieux résultats ne sont pas obtenus immédiatement et qu'ils dépendent de la motivation des patients à suivre un entraînement assidu et une rééducation appropriée. rurgicale immédiate passée, l'évaluation du nombre d'électrodes actives et la détermination des caractéristiques de stimulation efficace de chacune d'elles pour chaque patient permettent de mettre au point des procédures de traitement et d'encodage d'image spécifiques pour chaque patient.

La stimulation d'une ligne d'électrodes, par exemple, ne déclenche pas forcément la perception d'une ligne lumineuse car il faut tenir compte du positionnement de l'implant par rapport à l'axe visuel. Par ailleurs, même s'il est bien admis que les couches rétiniennes internes sont assez bien conservées y compris aux stades tardifs des rétinopathies pigmentaires, certains remaniements, même mineurs, peuvent perturber significativement la transmission des influx et engendrer des différences de perception majeures d'un patient à un autre. Différents protocoles de programmation peuvent également être proposés en fonction de la tâche visuelle à accomplir.

Nous ne mentionnons ici que quelques points qui jouent un rôle déterminant
En effet, une fois la période post-chi- dans le contrôle de la stimulation et la perception visuelle engendrée chez les patients. Bien plus importants que le nombre d'électrodes en jeu, le traitement et le codage de l'information visuelle (intensité, durée, fréquence, modalité de stimulation...) représentent la clé de voûte de cette approche de supplémentation ou substitution sensorielle.

De nombreux axes de recherche sont lancés pour optimiser l'utilisation concrète et pratique de tels implants, comme la miniaturisation des systèmes, l'amélioration des matériaux et de leur biocompatibilité...

Ces projets pourraient permettre dans les années à venir de mieux comprendre la formation du signal visuel pour tenter de lui suppléer artificiellement et de proposer des solutions de plus en plus performantes aux patients.

\section{Références}

Humayun MS, De Juan E Jr, Dagnelie G et al. « Visual perception elicited by electrical stimulation of retina in blind humans». Arch Ophthalmol. 1996;114:40-6. Chow AY, Chow VY, Packo KH et al. «The artificial silicon retina micro chip for the treatment of vision loss from retinitis pigmentosa ». Arch Ophthalmol. 2004; 122:460-9.

Humayun MS, Weiland JD, Fum GY et al. « Visual perception in a blind subject with a chronic microelectronic retinal prosthesis». Vision Res. 2003;43:2573-81.

Rizzo JF $3^{\text {rd }}$, Wyatt J, Loewenstein J et al. « Perceptual efficacy of electrical stimulation of human retina with a microelectrode array during short-term surgical trials ». Invest Ophthalmol Vis Sci. 2003;44:5362-9.

Rizzo JF 3rd, Wyatt J, Loewenstein J etal. « Methods and perceptual thresholds for short-term electrical stimulation of human retina with micro-electrode arrays ». Invest Ophthalmol Vis Sci. 2003;44:5355-61.

Salzmann J, Linderholm OP, Guyomard JLet al. « Subretinal electrode implantation in the $\mathrm{P} 23 \mathrm{H}$ rat for chronic stimulations». Dr J Ophthalmol 2006;90:1183-7.

Sommerhalder J, Oueghlani E, Bagnoud M, Leonards U, Safran AB, Pelizzone M. "Simulation of artificial vision: I. Eccentric reading of isolated words, and perceptual learning». Vision Res. 2003;43:269-83.

Remerciements

Les auteurs souhaitent exprimer leurs remerciements à l'ensemble du personnel du Centre d'investigation clinique pour leur contribution au succès de cette étude, en particulier à Céline Chaumette et Alexandre Leseigneur pour leur implication déterminante. 